\title{
Strong, Machinable, and Insulating Chitosan-Urea Aerogels: Toward Ambient Pressure Drying of Biopolymer Aerogel Monoliths
}

Natalia Guerrero-Alburquerque, Shanyu Zhao,* Nour Adilien, Matthias M. Koebel, Marco Lattuada, and Wim J. Malfait*

\begin{abstract}
Biopolymer aerogels are an emerging class of materials with potential applications in drug delivery, thermal insulation, separation, and filtration. Chitosan is of particular interest as a sustainable, biocompatible, and abundant raw material. Here, we present urea-modified chitosan aerogels with a high surface area and excellent thermal and mechanical properties. The irreversible gelation of an acidic chitosan solution is triggered by the thermal decomposition of urea at $80{ }^{\circ} \mathrm{C}$ through an increase in $\mathrm{pH}$ and, more importantly, the formation of abundant ureido terminal groups. The hydrogels are dried using either supercritical $\mathrm{CO}_{2}$ drying (SCD) or ambient pressure drying (APD) methods to elucidate the influence of the drying process on the final aerogel

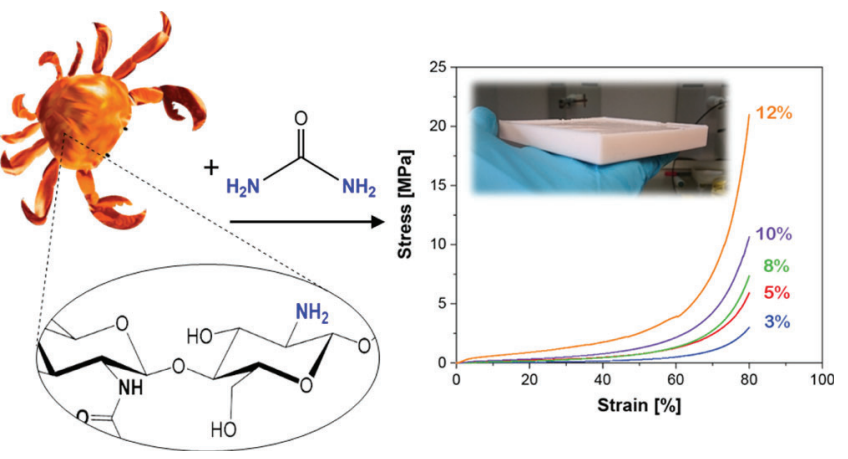
properties. The hydrogels are exchanged into ethanol prior to SCD, and into ethanol and then heptane prior to APD. The surface chemistry and microstructure are monitored by solid-state NMR and Fourier transform infrared spectroscopy, scanning electron microscopy, and nitrogen sorption. Surprisingly, large monolithic aerogel plates $\left(70 \times 70 \mathrm{~mm}^{2}\right)$ can be produced by APD, albeit at a somewhat higher density $\left(0.17-0.42 \mathrm{~g} / \mathrm{cm}^{3}\right)$. The as prepared aerogels have thermal conductivities of $\sim 24$ and $\sim 31 \mathrm{~mW} /(\mathrm{m} \cdot \mathrm{K})$ and surface areas of $160-170$ and $85-230 \mathrm{~m}^{2} / \mathrm{g}$, for SCD and APD, respectively. For a primarily biopolymer-based material, these aerogels are exceptionally stable at elevated temperature (TGA) and char and self-extinguish after direct flame exposure. The urea-modified chitosan aerogels display superior mechanical properties compared to traditional silica aerogels, with no brittle rupture up to at least $80 \%$ strain, and depending on the chitosan concentration, relatively high $E$-moduli $(1.0-11.6 \mathrm{MPa})$, and stress at $80 \%$ strain values $\left(\sigma_{80}\right.$ of $\left.3.5-17.9 \mathrm{MPa}\right)$. Remarkably, the aerogel monoliths can be shaped and machined with standard tools, for example, drilling and sawing. This first demonstration to produce monolithic and machinable, mesoporous aerogels from bio-sourced, renewable, and nontoxic precursors, combined with the potential for reduced production cost by means of simple APD, opens up new opportunities for biopolymer aerogel applications and marks an important step toward commercialization of biopolymer aerogels.
\end{abstract}

KEYWORDS: Mesoporous materials, Aerogel, Xerogel, Chitosan, Ureido, Ureylene, Urea

\section{INTRODUCTION}

Aerogels are highly porous and predominantly mesoporous solids with exceptional properties and a rapidly growing market, particularly silica aerogels as thermal superinsulation materials. ${ }^{1-4}$ Traditionally, aerogels are prepared by extracting a gel's pore fluid with supercritical $\mathrm{CO}_{2}$ drying (SCD) to circumvent the strong capillary forces that occur at liquid-gas interfaces during solvent evaporation. However, supercritical drying is a high-pressure process that requires nontrivial equipment and is limited as its scaling requires high cost. Ambient pressure drying (APD) is a viable alternative to produce high-quality silica aerogels if the gel surfaces have been hydrophobized, ${ }^{5}$ but is generally not feasible for hydrophilic aerogel systems because of excessive shrinkage and structural collapse.
Biopolymer aerogels are a potentially more sustainable alternative to silica aerogels, and a wide variety of precursors are available, mostly polysaccharides (e.g. cellulose, alginate, chitin and chitosan, pectin, and starch), but also proteins and lignins. ${ }^{6}$ Chitin and its deacetylated product chitosan are of particular interest because they are derived from shrimp and crab shell wastes, of which six to eight million tonnes are produced per annum. ${ }^{7,8}$ In addition, chitosan's amino groups, from the (deacetylated) D-glucosamine units, can impart their 


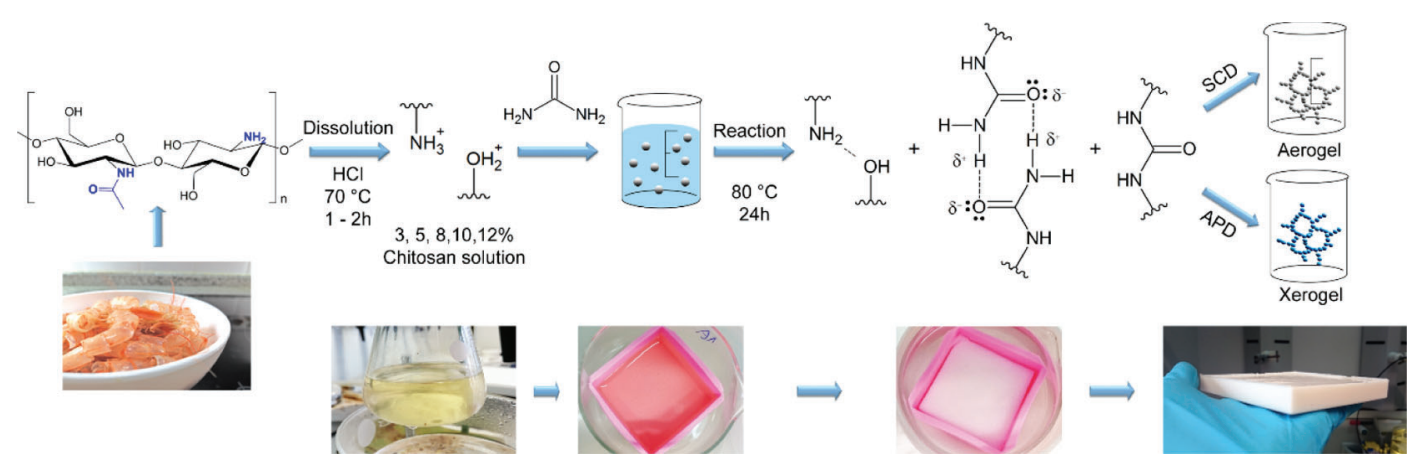

Figure 1. Chitosan aerogel synthesis and the gelation mechanism.

corresponding aerogels with specific functionality, including $\mathrm{CO}_{2}$ sorption, ${ }^{9,10}$ metal ion absorption, ${ }^{9,11,12}$ dye absorption, ${ }^{13,14}$ trapping of contaminants, ${ }^{15,16}$ catalysis, ${ }^{17-23}$ reinforcement of other (e.g. silica) aerogels, ${ }^{24}$ and chitosan derivatives, $^{25-27}$ and are used as an encapsulation matrix for small molecules and nanoparticles, ${ }^{25,28-30}$ in addition to their traditional thermal insulation applications. ${ }^{13,31-33}$

The gelation of chitosan solutions can be induced either by means of (i) physical mechanisms, for example by a $\mathrm{pH}$ or temperature change, (ii) by coagulation in nonsolvents, or iii) through (covalent) cross-linking with reactive cross-linkers. The physical gelation mechanism uses $\mathrm{pH}$, sometimes combined with temperature, to induce gelation. Dripping an acidic aqueous solution of chitosan into an alkaline solution causes coagulation because of the $\mathrm{pH}$ inversion effect ${ }^{34,35}$ and also a $\mathrm{pH}$-lowering gelation through pressurized $\mathrm{CO}_{2}$ induces chitosan gelation, ${ }^{36}$ but the gelation is reversible and chitosan can be dissolved again upon acidification. ${ }^{37,38}$ Physical gelation can also be induced by increasing the temperature of a chitosan solution, buffered to a neutral $\mathrm{pH}$ by $\beta$-glycerolphosphate. ${ }^{39}$ Increased temperatures were also used to cross-link hemicellulose citrate with chitosan at low $\mathrm{pH} .{ }^{40}$ Another physical gelation strategy involves coagulation of chitosan in a nonsolvent ${ }^{41}$ in a process akin to that for the production of regenerated cellulose aerogels. ${ }^{42}$ Immersion in ethanol facilitated the gelation of polysaccharide aerogels without further addition of cross-linkers, but the resulting aerogels had limited mechanical strength. ${ }^{43}$ Even for cross-linked chitosan gels, solvent-chitosan interactions, including the solvent exchange into $\mathrm{CO}_{2}$ during supercritical drying, strongly affect the microstructure and properties. ${ }^{44}$

Although chitosan is a nontoxic, renewable precursor, gels and aerogels are often produced by cross-linking the amino groups with "non-friendly" and often toxic cross-linkers, particularly formaldehyde, ${ }^{13,15,31,32,45}$ glutaraldehyde, ${ }^{15,16,46}$ glyoxal, $^{15}$ and diisocyanates, ${ }^{27}$ which negate some of the benefits of using a sustainable, bio-sourced precursor. However, there are effective techniques to eliminate the remains of these cross-linkers, for example the removal of glutaraldehyde using a mixture of $\mathrm{CO}_{2}$ and ethanol during supercritical drying. ${ }^{47}$ In addition, more benign cross-linkers are available, but have been applied only sporadically to the synthesis of chitosan aerogels. Cross-linking amino groups with $\mathrm{CO}_{2}$ has not yet been applied to chitosan, but has been demonstrated successfully to produce polyurea from synthetic polyamines, either in pressurized reactors, ${ }^{48}$ or by bubbling $\mathrm{CO}_{2}$ through a polyallylamine solution. ${ }^{49}$ Urea is a possible cross-linker for amino groups that has been mostly overlooked in the latest non-isocyanate polyurea-polyurethane and aerogel literature. Before the advent of isocyanates, urea was the preferred cross-linker for polycondensation of polyurea, with a rich patent literature going back as far as the $1930 \mathrm{~s}^{50-52}$ Typically, large diamines or alkanolamines were reacted with urea in bulk or in solution at a relatively high temperature $\left(\sim 200{ }^{\circ} \mathrm{C}\right)$ through the reaction

$$
2 \mathrm{R}-\mathrm{NH}_{2}+\mathrm{H}_{2} \mathrm{NCONH}_{2} \rightarrow \mathrm{R}-\mathrm{NHCONH}-\mathrm{R}+2 \mathrm{NH}_{3}
$$

A recent study describes the urea-chitosan combination to produce gels, ${ }^{53}$ working with urease to accelerate the hydrolysis of urea. ${ }^{54}$ Very recently, Ganesan et al. demonstrated a facile method to synthesize ureido-modified chitosan gels and aerogels. ${ }^{55}$

In this study, we investigate the preparation of chitosan aerogels with a high surface area and mesoporosity, and excellent mechanical and thermal properties using a urea modification. Both supercritical and, surprisingly, ambient pressure drying can yield large, defect-free monolithic aerogel plates. The gelation mechanism is probed using Fourier transform infrared (FTIR) and solid-state NMR spectroscopic data, which-in combination with complimentary characterization techniques-allows systematically mapping out the effect of chitosan concentration and drying conditions on shrinkage, density, microstructure, thermal conductivity, and mechanical properties.

\section{EXPERIMENTAL SECTION}

2.1. Materials. Chitosan from crab shells with a high viscosity (>400 MPa s for $1 \%$ in acetic acid $@ 20{ }^{\circ} \mathrm{C}$ ), a high molecular weight (HW) $\left(310,000-375,000 \mathrm{~g} \mathrm{~mol}^{-1}\right)$, and a degree of deacetylation (DDA) of $77 \%$, urea BioXtra, a $320 \mathrm{~g} \mathrm{~mol}^{-1}$ cetyltrimethylammonium chloride (CTAC) solution, and the $\mathrm{HCl}$ ACS Reagent ( $\mathrm{HCl} \mathrm{37 \% )}$ were purchased from Sigma Aldrich (Switzerland). Ethanol (F25-AFMEK ethanol denatured with $2 \%$ methyl ethyl ketone) was sourced from Alcosuisse, Switzerland. Heptane (isomer mixture, UN 1206) was obtained from Brenntag (Switzerland). Deionized water was used for dilutions. All reagents and solvents were used as received without further purification.

2.2. Synthesis of Chitosan-Urea Aerogels. Let us explain the aerogel synthesis using the example of a typical sample prepared from the $10 \% \mathrm{~m} / \mathrm{v}$ chitosan solution (Figure 1); preparations for other concentrations are summarized in Table S1. Aerogels were prepared by dissolving $4 \mathrm{~g}$ chitosan in $40 \mathrm{~mL}$ of a $1.12 \mathrm{M} \mathrm{HCl}$ aqueous solution under constant stirring at $70{ }^{\circ} \mathrm{C}$ in an oil bath for approximately $2 \mathrm{~h}$. The resulting, somewhat viscous, yellowish-transparent solution was taken out of the oil-bath and left to cool at room temperature for 5 min under stirring. After the dissolution of $9 \mathrm{~g}$ of urea, a very minor amount of surfactant ( $30 \mu \mathrm{L} \mathrm{CTAC)}$ was added to help eliminate bubbles. The solution was then centrifuged at $6000 \mathrm{rpm}$ for $3 \mathrm{~min}$ to eliminate residual trapped air and possible insoluble solids, which were removed by decantation. The chitosan-urea solution was 


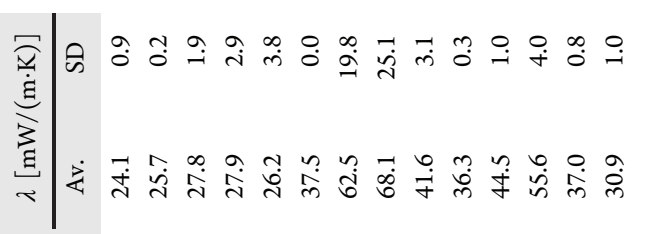

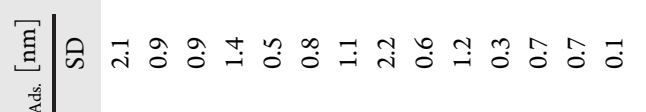

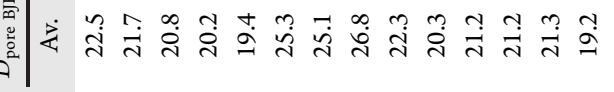

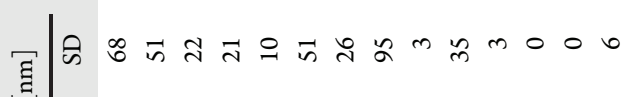

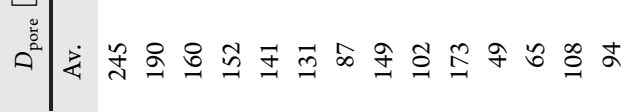

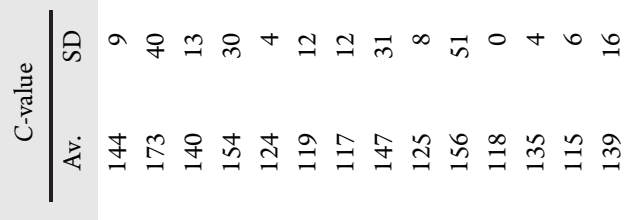

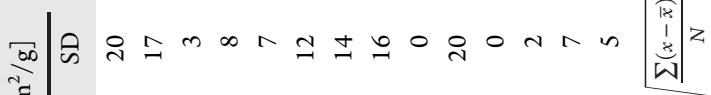

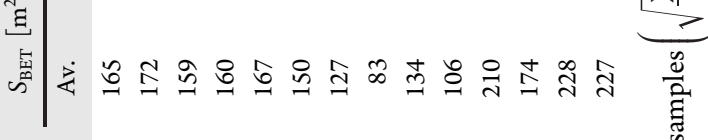

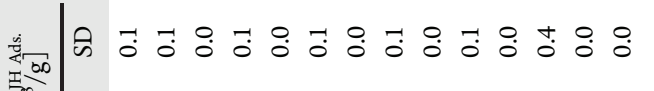

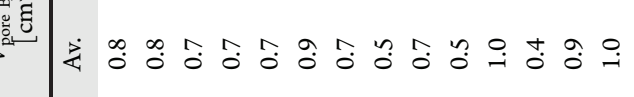

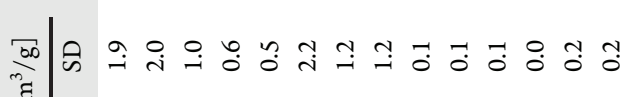

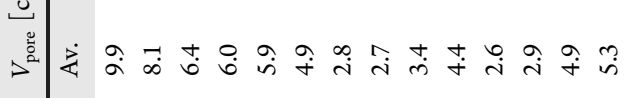

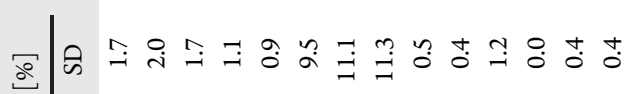

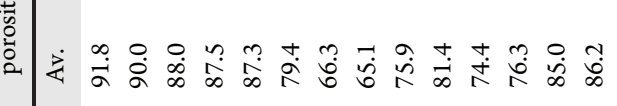

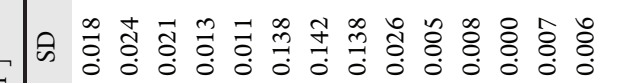

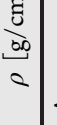

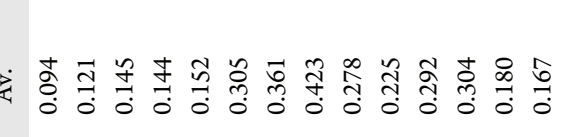

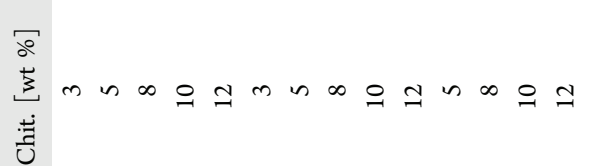

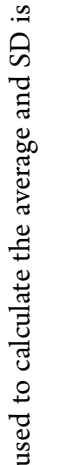

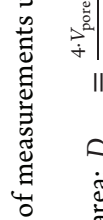

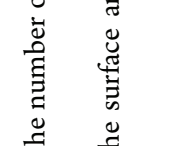




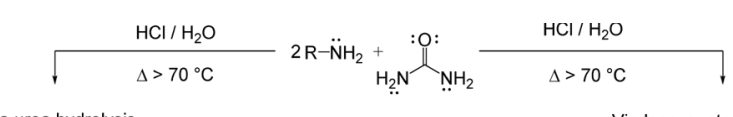

Via urea hydrolysis

Via Isocyanate Intermediate
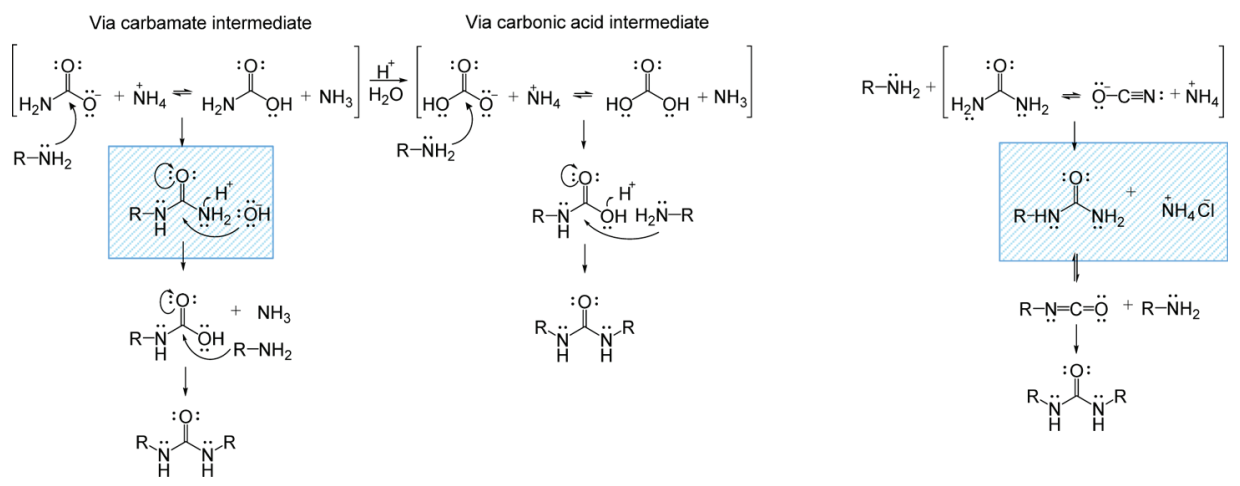

Figure 2. Possible reaction mechanisms for the formation of end-capping ureido groups (dominant species) and, possibly, cross-linking ureylene groups (minor species). The full decomposition of urea with water into $\mathrm{CO}_{2}$ and $\mathrm{NH}_{3}$ is not shown.

poured into square silicone molds $\left(25 \mathrm{~mL}\right.$ of sol in $5 \times 5 \times 3 \mathrm{~cm}^{3}$ for $\mathrm{SCD}, 60 \mathrm{~mL}$ of sol in $9 \times 9 \times 3 \mathrm{~cm}^{3}$ for APD) and placed into a larger plastic polypropylene sample storage container. Gelation and aging took place at $80^{\circ} \mathrm{C}$ for $24 \mathrm{~h}$. Over the course of the gelation and aging process, the $\mathrm{pH}$ value changed from $\sim 0$ to 7 .

The gels intended for SCD were exchanged into ethanol 3 times (gel/solvent ratio $1: 10,65{ }^{\circ} \mathrm{C}, 24 \mathrm{~h}$ each) and then dried from supercritical $\mathrm{CO}_{2}$ (SCD) in an autoclave (4334/A21-1 Separex, France). The ethanol pore fluid was exchanged into liquid $\mathrm{CO}_{2}$ at $\sim 7$ ${ }^{\circ} \mathrm{C}$ in a batch type autoclave. After several flushing steps with liquid $\mathrm{CO}_{2}$ for $\sim 8 \mathrm{~h}$, the ethanol was exchanged with $\mathrm{CO}_{2}$. The chamber was isolated from the $\mathrm{CO}_{2}$ supply and the temperature was increased to $50{ }^{\circ} \mathrm{C}$ over the course of about $4 \mathrm{~h}$, leading to an increase in pressure to typically 150 bar. Subsequently, the supercritical $\mathrm{CO}_{2}$ autoclave was depressurized within $1 \mathrm{~h}$ and allowed to cool to room temperature prior to removing the samples. A second set of samples was prepared by ambient pressure drying. Here, the as prepared gels were washed thrice with ethanol (gel: solvent ratio $1: 10,65^{\circ} \mathrm{C}, 24 \mathrm{~h}$ per washing step), and then exchanged into heptane 3 times (gel: solvent ratio $1: 10,25^{\circ} \mathrm{C}, 24 \mathrm{~h}$ per exchange step). The samples were then allowed to dry at ambient pressure in open containers, either at room temperature or at $65{ }^{\circ} \mathrm{C}$.

2.3. Characterization. The linear shrinkage of the gels for each of the processing steps was monitored by measuring the edge length of the square samples with a caliper, $\left(L-L_{0}\right) / L_{0}$, where $L$ is the length of the sample after each processing step and $L_{0}$ is the length of the sample container. The size of the relatively weak hydrogels could not be determined accurately, but the shrinkage after gelation and aging was minimal (between 0 and 2\%) for all hydrogels. The apparent bulk density was calculated from the weight and the dimensions of the regularly shaped monolithic samples (square plates and cylinders). The skeletal density $\left(\rho_{\text {skeletal }}\right)$ was measured using a helium pycnometry (AccuPyc II 1340). No degassing was performed prior to the density measurements. The pore volume $\left(V_{\text {pore }}\right)$ along with the porosity $(\% \phi)$ was calculated from the bulk and skeletal density of the aerogel eqs 1 and 2.

$$
\begin{aligned}
& V_{\text {pore }}=\frac{1}{\rho_{\text {bulk }}}-\frac{1}{\rho_{\text {skeletal }}} \\
& \% \phi=\left(1-\frac{\rho_{\text {bulk }}}{\rho_{\text {skeletal }}}\right) \cdot 100
\end{aligned}
$$

Nitrogen sorption isotherms were measured at liquid nitrogen temperature on a Micromeritics Triflex instrument after degassing the samples at $3.3 \times 10^{-2} \mathrm{mbar}$ and $80^{\circ} \mathrm{C}$ for $40 \mathrm{~h}$. The specific surface areas $\left(S_{\mathrm{BET}}\right)$ were calculated from 4 to 6 data points in the linear range of $P / P_{0}$ between 0.05 and 0.3 using the BET (Brunauer-
Emmett-Teller) method. The $S_{\mathrm{BET}}$ and $C$-values are listed in Tables 1 and S10. The average pore diameter was calculated from the pore volume and surface area assuming cylindrical pores [eq 3], rather than the classical BJH (Barrett-Joyner-Halenda) model for analysis, ${ }^{56}$ which for the aerogel is affected by mechanical deformation in the desorption branch of the capillary condensation range. ${ }^{57}$ The precision of the BET surface area is approximately $10 \mathrm{~m}^{2} / \mathrm{g}$, but the accuracy may be lower (around $50 \mathrm{~m}^{2} / \mathrm{g}$ ) because of the model dependencies.

$$
D_{\text {pore }}=\frac{4 \cdot V_{\text {pore }}}{S_{\mathrm{BET}}}
$$

Scanning electron microscopy (SEM) images were recorded with an FEI Nova NanoSEM 230 (FEI, Hillsboro, Oregon, USA) at an accelerating voltage between 5 and $10 \mathrm{kV}$ and a working distance of around $5 \mathrm{~mm}$. The samples were fixed using carbon pads and coated with Pt at a nominal thickness of $20 \mathrm{~nm}$. Note that the actual thickness of the coating on the aerogel surfaces is much lower because of the extreme topography.

Solid-state NMR spectra were acquired with a Bruker Avance III system equipped with a wide-bore $9.4 \mathrm{~T}$ magnet, corresponding to Larmor frequencies of $400.2 \mathrm{MHz}$ for ${ }^{1} \mathrm{H}$ and $100.6 \mathrm{MHz}$ for ${ }^{13} \mathrm{C}$, using $7 \mathrm{~mm}$ diameter zirconia rotors and at a magic angle spinning (MAS) rate of $4 \mathrm{kHz} \cdot{ }^{1} \mathrm{H}-{ }^{13} \mathrm{C}$ cross-polarization spectra were acquired using a recycle delay of $3 \mathrm{~s}$ and a relatively long contact time of $2 \mathrm{~ms}$ to minimize the dependency of the relative spectral intensities on the hydrogen-carbon distance. FTIR spectra were collected with a Bruker Tensor 27 FTIR spectrophotometer with a scan range from 400 to $4000 \mathrm{~cm}^{-1}$. Spectra were collected in attenuated total reflectance mode (ATR) using a diamond crystal.

The thermal conductivity $(\lambda)$ was measured with a custom-built guarded hot plate device (protected zone: $50 \times 50 \mathrm{~mm}^{2}$, measuring zone: $25 \times 25 \mathrm{~mm}^{2}$ ) specifically designed to measure small low $\lambda$ samples (Figure S1). ${ }^{58}$ The thermal conductivity was determined from monolithic square-shaped plates $(\mathrm{SCD}$ plates around $50 \times 50 \times$ $10 \mathrm{~mm}, \mathrm{APD}$ plates around $\left.70 \times 70 \times 10 \mathrm{~mm}^{3}\right)$ after preconditioning at $30 \%$ relative humidity $(\mathrm{RH})$ and $25{ }^{\circ} \mathrm{C}$ for at least $24 \mathrm{~h}$. Thermogravimetric analysis was carried out using a Netzsch TGA 209 $\mathrm{F} 1$ instrument in reconstituted air $\left(80 \% \mathrm{v} / \mathrm{v} \mathrm{N}_{2}\right.$ and $\left.20 \% \mathrm{v} / \mathrm{v} \mathrm{O}_{2}\right)$, from 30 to $900{ }^{\circ} \mathrm{C}$ with a heating rate of $20^{\circ} \mathrm{C} / \mathrm{min}$.

The mechanical properties were evaluated on cylindrical samples (15 mm diameter, $20-30 \mathrm{~mm}$ high) using a universal mechanical testing setup (Zwick/Z010, Zwick/Roell, Germany) to apply uniaxial compression with a $10 \mathrm{kN}$ force transducer (KAP-S, AST Gruppe $\mathrm{GmbH}$, Germany) at a rate of $1 \mathrm{~mm} / \mathrm{min}$ up to $80 \%$ strain in a controlled environment $\left(23{ }^{\circ} \mathrm{C}\right.$ and $\left.50 \% \mathrm{RH}\right)$. The elastic moduli were calculated from the linear region of the stress-strain curves (typically between 1 and 3\% strain). 

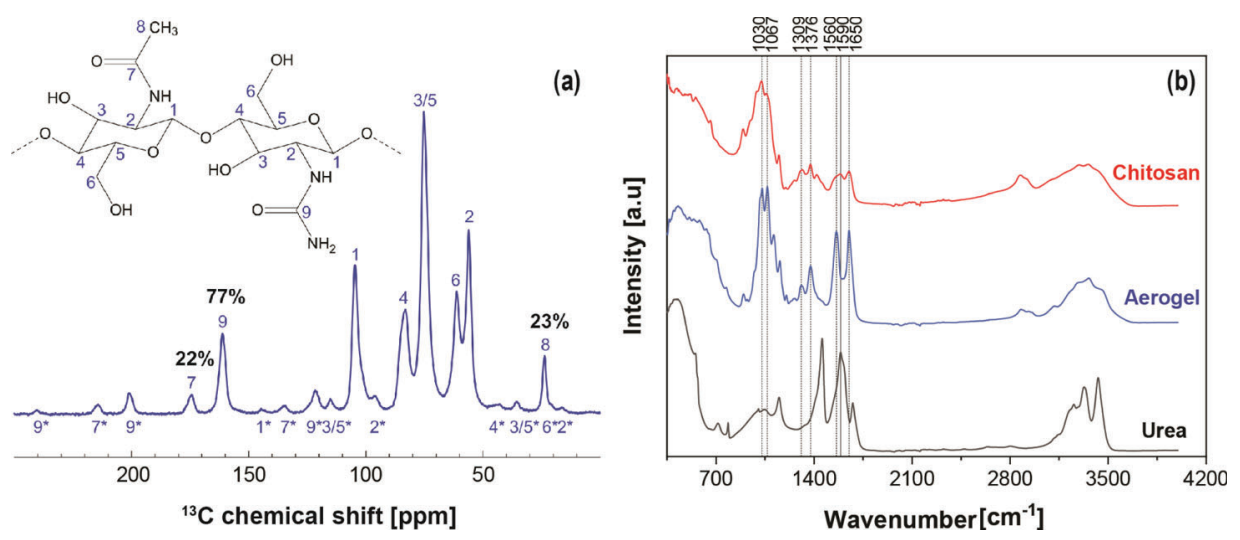

Figure 3. (a) Solid-state CP-MAS ${ }^{1} \mathrm{H}-{ }^{13} \mathrm{C}$ NMR spectrum of the chitosan-urea aerogel (GA067). Peak assignments are illustrated with a fragment of chitosan; peaks labeled with an asterisk $(*)$ are spinning sidebands. The relative intensity of the acetyl groups (C7/C8), including spinning sidebands and normalized to the total intensity of the $\mathrm{C} 1$ band, amounts to $22 / 23 \%$. The relative intensity of ureido (C9) groups is $77 \%$. (b) ATR-FTIR spectra of pure chitosan, aerogel, and urea.

\section{RESULTS AND DISCUSSION}

\subsection{Parameters Affecting the Gelation and Reaction} Mechanism. The main parameters that affect gelation are temperature and the urea concentration: gelation only occurs for reaction temperatures of at least $80{ }^{\circ} \mathrm{C}$ and urea/Dglucosamine molar ratios of at least 4/1 (Tables S2 and S3). The effect of temperature is particularly striking: no gelation is observed at $70^{\circ} \mathrm{C}$, even after $48 \mathrm{~h}$, whereas a strong gel that could be processed to an aerogel was obtained after just $5 \mathrm{~h}$ at $80{ }^{\circ} \mathrm{C}$. The $80^{\circ} \mathrm{C}$ required for gelation is consistent with the decomposition temperature of urea in the aqueous solution for the two possible mechanisms, through hydrolysis s $^{5-64}$ and/or through an isocyanate intermediate (Figure 2). ${ }^{65,66}$ Note also that the thermal decomposition of urea represents the onset of the formation of ammonia, is responsible for the neutralization of the acidic sol. Mechanistically, we are not able to discern which of the proposed reaction mechanisms dominates during the aerogel synthesis, primarily because of the short-lived nature and the corresponding small effective concentrations of the intermediates. The results discussed above were obtained for a chitosan concentration of $10 \% \mathrm{~m} / \mathrm{v}$, but for the optimum conditions $\left(80^{\circ} \mathrm{C}\right.$, urea $/ \mathrm{D}$-glucosamine $\left.=6 / 1,24 \mathrm{~h}\right)$, gelation was observed for all investigated chitosan loadings $(3,5,8,10$, and $12 \% \mathrm{~m} / \mathrm{v}$ ). The effect of protonation of chitosan in the solution, that is the concentration of $\mathrm{HCl}$, was also investigated (Table S4), but all data in the remainder of this manuscript correspond to a degree of protonation of $180 \%$. Note that chitosan depolymerizes under these conditions through the hydrolysis of the glycosidic bonds, ${ }^{67-69}$ therefore dissolution temperatures and times need to be kept constant to obtain consistent results. The dissolution of different concentrations of HW chitosan in different acids was also investigated, without success (Table S6). Note that the experiments above were carried out with chitosan with a high molecular weight $(310,000-375,000 \mathrm{~g} / \mathrm{mol})$ and that no gelation was observed with chitosan with medium $(190,000-310,000 \mathrm{~g} / \mathrm{mol})$ and low $(50,000-190,000 \mathrm{~g} / \mathrm{mol})$ molecular weights.

3.2. Aerogel Chemistry and Structure Formation. 3.2.1. Solid-State NMR Spectroscopy. The ${ }^{1} \mathrm{H}-{ }^{13} \mathrm{C}$ CP MAS NMR spectrum of the chitosan-urea aerogel displays all the signatures expected for chitosan with a high DDA (Figure 3a). Cross-polarization spectra are not inherently quantitative because the relative peak intensities are modified by variable relaxation times and cross-polarization efficiencies. Nevertheless, the reasonable agreement between the nominally expected (1.0/1.0/2.0/1.0/1.0) and measured (1.0/1.2/2.3/ 1.1/0.9) peak areas for $\mathrm{C} 1, \mathrm{C} 2, \mathrm{C} 3+\mathrm{C} 5, \mathrm{C} 4$, and C6 (Table S7) indicates that, for these samples and under these experimental conditions, the peak areas provide a reasonable approximation of the carbon abundances. The normalized intensities of the $\mathrm{C} 7(0.22)$ and $\mathrm{C} 8(0.23)$ bands indicate a degree of acetylation of around $23 \%$. The normalized intensity of 0.77 of the $\mathrm{C} 9$ band, assigned to the $\mathrm{C}=\mathrm{O}$ group of endcapping ureido groups, indicates that, within uncertainty, all of the deacetylated amino groups have reacted with urea to form ureido groups, that is few to no primary amino groups remain on the chitosan backbone. The presence of abundant endcapping ureido groups and the absence in the spectra of crosslinking urea groups are not unexpected. Because of the large urea excess at which the reaction is carried out (6:1 molar urea/D-glucosamine ratio), only $3 \%$ of ureylene cross-links would be expected based on a simple simulation that assumes equal kinetic rate constants for the end-capping (first step) and cross-linking (second step) reactions (Figure S5). However, a parallel ongoing study in our laboratory on the reaction between ethanolamine and urea indicates that the probability of the ureylene cross-linking reaction is much lower than that of the ureido end-capping reaction. Thus, the $3 \%$ estimate represents an upper bound concentration of cross-links. Because of the high molecular weight of chitosan, possible cross-links could still be expected to have a substantial effect on gelation and structure formation, even at a (very) low concentration. In other words, only a few cross-links would be needed to increase viscosity and promote gelation.

3.2.2. FT-IR Spectroscopy. The FTIR spectrum of the ureamodified chitosan aerogel closely resembles that of neat chitosan (Figure 3b), apart from distinct changes in the intensity and peak position in the $1550-1700 \mathrm{~cm}^{-1}$ region. Neat chitosan has two peaks in this region, one at $1590 \mathrm{~cm}^{-1}$ was assigned to the $-\mathrm{NH}_{2}$ groups and one at $1650 \mathrm{~cm}^{-1}$ related to the acetyl group, that is $-\mathrm{NHC}(\mathrm{O}) \mathrm{CH}_{3}$. The vibrational band of the $-\mathrm{NH}_{2}$ groups at $1590 \mathrm{~cm}^{-1}$ is absent in the chitosan aerogel spectrum, but the urea-modified chitosan aerogels display two peaks in this region, at 1650 and 1560 $\mathrm{cm}^{-1}$, typical for amide I and amide II vibrations, that is from the $-\mathrm{NHC}(\mathrm{O}) \mathrm{NH}_{2}$ groups, that are too intense to arise entirely from the acetyl amide bands from the original 

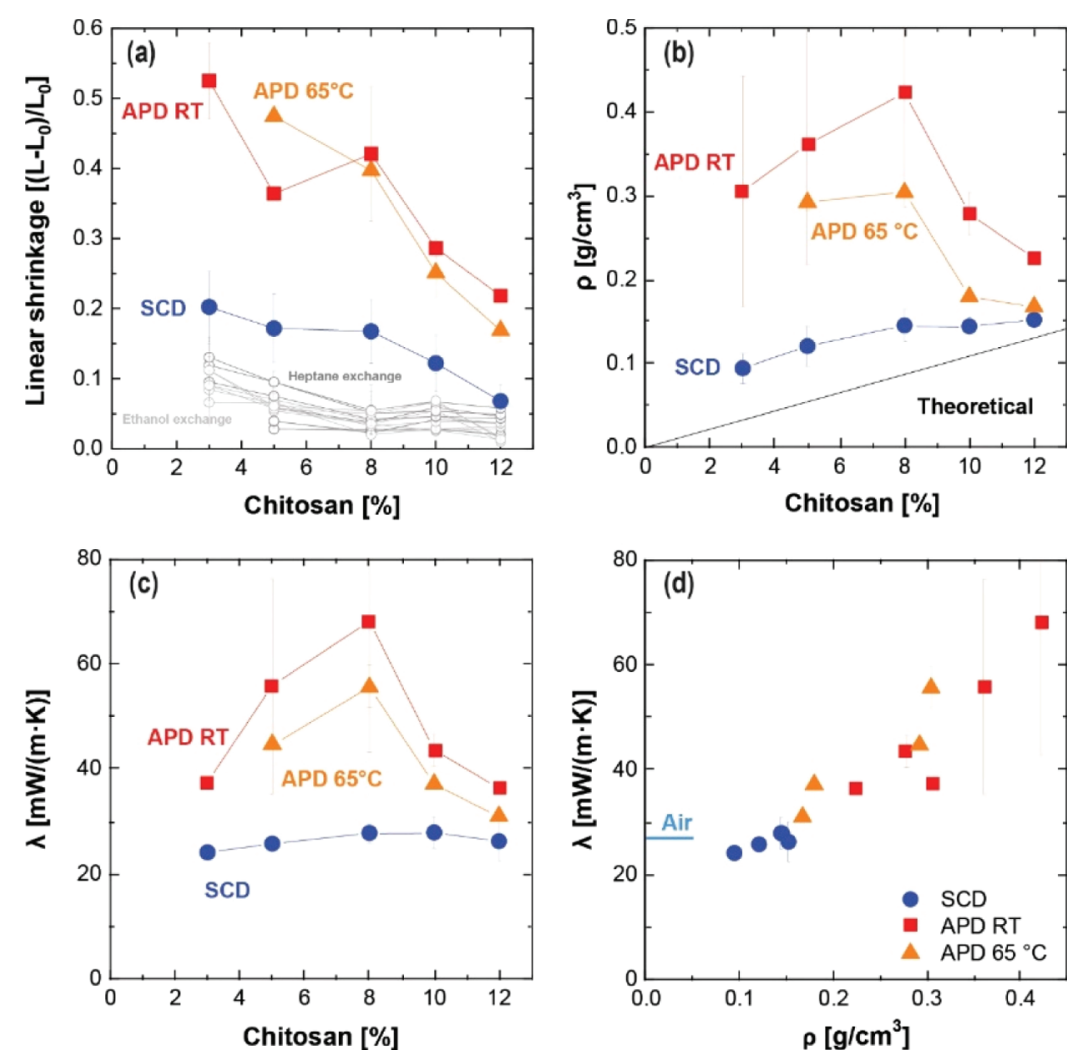

Figure 4. (a) Linear shrinkage as a function of chitosan concentration. (b) Density dependence on the chitosan concentration. (c) Thermal conductivity versus chitosan concentration. (d) Thermal conductivity versus density.

chitosan. The experimental peak positions should not be overinterpreted as the bands are the sum of at least two overlapping sets of peaks (residual acetyl and newly formed ureido groups). Nevertheless, the relatively low frequency of the carbonyl stretching vibrations $\left(1650 \mathrm{~cm}^{-1}\right)$ is indicative of moderate to strong hydrogen bonding as a result of the delocalization of electrons in the $\pi$-bonds. ${ }^{70}$ Compared to the neat chitosan spectrum, the peak at $1422 \mathrm{~cm}^{-1}$ related to $\mathrm{CH}_{2}$ bending disappears in the aerogel spectrum and the peak assigned to $\mathrm{C}-\mathrm{H}$ bending at $1376 \mathrm{~cm}^{-1}$ and to a lesser extent also the peak at $1309 \mathrm{~cm}^{-1}$ from $\mathrm{C}-\mathrm{N}$ stretching (amide III) are more intense. The overlapping vibrational bands ascribed to $\mathrm{C}-\mathrm{O}$ at 1030 and $1067 \mathrm{~cm}^{-1}$ display two well-defined peaks in the aerogel spectrum. The vibrational bands of $\mathrm{O} 3-\mathrm{H}$ and O6-H appear at relatively low frequencies in the aerogel spectrum (3361 and $3453 \mathrm{~cm}^{-1}$, respectively), indicating relatively strong hydrogen bonding. The complete peak assignments are listed in Table S8.

3.2.3. Structure Formation. The change from a clear solution to a white gel during aging and gelation (at $80{ }^{\circ} \mathrm{C}$ ) indicates that larger aggregates that scatter visible light have formed during gelation, that is before solvent exchange and drying. Aggregation can be related to multiple effects (Figure 1 ), namely (i) ammonia released during urea decomposition neutralizes the acid and reduces chitosan solubility, ${ }^{60}$ (ii) the grafting of ureido groups onto the chitosan backbone, which changes the solvent-chitosan and chitosan-chitosan interactions, and enables the establishment of a "ureido" based hydrogen bonding network, and (iii) the actual cross-linking of chitosan by ureylene moieties. The change in $\mathrm{pH}$ alone is not sufficient to explain the structure formation here, because chitosan gelation through the addition of base is generally reversible, ${ }^{37,49,71}$ which is not the case in our system: no dissolution of mass was observed after immersion of the ureamodified hydrogels in a $\mathrm{pH} 2 \mathrm{HCl}$ solution for $24 \mathrm{~h}$. In addition, gels produced by base addition to acidic chitosan solutions are mechanically much weaker than those prepared here. Thus, the microstructure and properties of the gels and aerogels produced here must be strongly linked to either or both of the remaining mechanisms, that is grafting of ureido groups and cross-linking with ureylene groups.

3.3. Shrinkage, Density, Humidity Uptake, Thermal Stability, and Thermal Conductivity. 3.3.1. Shrinkage and Density. The importance of shrinkage of (bio)polymer aerogels during processing has been highlighted recently. ${ }^{72}$ For all investigated processing steps (solvent exchanges, drying), there is a clear, inverse correlation between the chitosan concentration and linear shrinkage (Figure 4a), as is typical for most aerogel systems and simply because the increased mechanical strength of more concentrated gels enables them to better withstand the stresses during processing. Compared to other biopolymer aerogel systems, ${ }^{72}$ the solvent exchange into ethanol and heptane leads to only a moderate linear shrinkage (between 2 and $12 \%$ for $3 \% \mathrm{~m} / \mathrm{v}$ chitosan, and between 2 and $4 \%$ for $12 \% \mathrm{~m} / \mathrm{v}$ chitosan), despite the very low solubility of chitosan in ethanol and heptane. We interpret this as further proof that the microstructure/aggregation of chitosan occurred before washing and solvent exchange steps, that is during gelation and aging. Shrinkage is more pronounced after drying. For SCD, the higher linear shrinkage $(20 \%)$ at low concentrations results in a relatively flat dependence of density on the chitosan concentration, but the lowest densities are observed for the lowest chitosan concentrations (Figure 4b). In the case of 

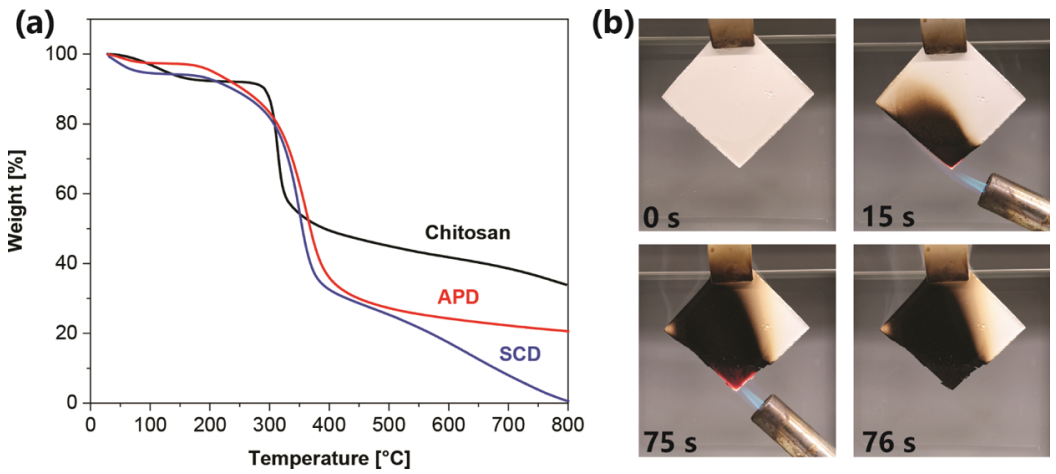

Figure 5. (a) Thermogravimetry of neat chitosan and chitosan-urea aerogels (SCD and APD, $10 \% \mathrm{~m} / \mathrm{v}$ chitosan); the analysis was conducted in reconstituted air. (b) Fire behavior of the APD chitosan-urea aerogel (10\% m/v chitosan).

APD, we see much higher shrinkage and also the dependence of shrinkage on the chitosan concentration is even more extreme. The apparent density dependence is a complex function of the chitosan concentration with the lowest APD densities being observed for the highest chitosan concentrations, contrary to the SCD samples. The faster drying at higher APD temperatures reduces the time for which the gels are exposed to drying-induced capillary forces. The lower densities after APD at $65{ }^{\circ} \mathrm{C}$, compared to room temperature, seem to confirm this hypothesis.

3.3.2. Thermal Conductivity. The thermal conductivity $\lambda$ (Figure 4c) strongly correlates with aerogel density: The SCD and $\mathrm{APD}\left(\mathrm{RT} / 65{ }^{\circ} \mathrm{C}\right)$ data define a single monotonously increasing function of aerogel density (Figure $4 \mathrm{~d}$ ). No minimum in thermal conductivity is observed at intermediate densities, as is typical for many aerogel systems, ${ }^{1,73}$ but the lowest thermal conductivity is observed for the lowest density samples. This indicates the important contributions of the solid conduction in shaping the density dependence of thermal conductivity over the investigated density range $(0.094-0.423$ $\left.\mathrm{g} / \mathrm{cm}^{3}\right)$. Thermal conductivities as low as $24.1 \mathrm{~mW} /(\mathrm{m} \cdot \mathrm{K})$ are reached for the lowest density SCD-dried aerogels (Table 1) and even lower conductivities were measured for aerogels processed at $90{ }^{\circ} \mathrm{C}(22.1 \mathrm{~mW} /(\mathrm{m} \cdot \mathrm{K})$, Table S3). These values are well below that of standing air $(26 \mathrm{~mW} /(\mathrm{m} \cdot \mathrm{K}))$ and much lower than for conventional insulation materials (e.g. mineral wool or EPS with $\sim 33 \mathrm{~mW} /(\mathrm{m} \cdot \mathrm{K}))$, indicating that the gas phase conduction is at least partially suppressed through the Knudsen effect because of the mesoporous network. Presumably, the gas phase conduction is even lower for the higher density aerogels with their smaller pores, but this effect is masked by the higher solid-phase conduction. The APD aerogels display higher densities and thermal conductivities, but still in line with those of conventional insulation materials. The lower densities for APD at $65{ }^{\circ} \mathrm{C}$ (compared to room temperature) lead to lower thermal conductivities: a value of $30.9 \mathrm{~mW} /(\mathrm{m} \cdot \mathrm{K})$ is achieved for the lowest density APD-dried aerogel (Table 1).

3.3.3. Moisture Uptake and Thermal Stability. The chitosan-urea aerogels are hydrophilic and display very substantial water uptake, even at low $\mathrm{RH}$ (Figure S2). For example, the $10 \%$ SCD chitosan samples display a humidity uptake of $2.4,6.1$, and $6.3 \% \mathrm{w} / \mathrm{w}$ after exposure to $30 \% \mathrm{RH}$ for $1 \mathrm{~h}, 1$ day, and 14 days, respectively. The APD samples show a similar behavior. The relatively high moisture uptake is expected to negatively affect the thermal conductivity as it is known to increase through water uptake. ${ }^{74}$ Hydrophobization of the chitosan aerogels may help to decrease the thermal conductivity and/or reduce its dependence on $\mathrm{RH}$, but only a single study has reported on the hydrophobization of mesoporous chitosan aerogels. ${ }^{32,45}$ Generally, the durable hydrophobization of biopolymer-based aerogels remains a big challenge for the community. In addition, most hydrophobization protocols seem to only improve the (liquid) water-contact angle while leaving humidity uptake relatively unaffected. $^{75}$

The humidity uptake is reflected in the weight loss (2.6$5.5 \%)$ upon drying as seen during thermogravimetry between 50 and $150{ }^{\circ} \mathrm{C}$ for all the investigated materials: chitosan starting material, SCD, and APD aerogels (Figure 5a). A more pronounced, rapid loss of mass of 65 to $70 \%$ is observed with a maximum rate at $\sim 350{ }^{\circ} \mathrm{C}$, which is higher than that for neat chitosan $\left(\sim 315^{\circ} \mathrm{C}\right)$, but with a lower onset temperature (200 ${ }^{\circ} \mathrm{C}$ ). The good high temperature stability as observed by TGA is consistent with the aerogel's behavior when exposed to a flame (Figures 5b, S4, S5). Both the SCD and APD aerogels char and carbonize rather than burn when exposed to a flame, and retain their structural integrity throughout. Once the heat source is removed, the glow disappears immediately. The fireretarding properties of chitosan are well known, particularly in combination with phosphate chemistry. ${ }^{76,77}$ The nature of the degradation products after flame exposition was not investigated here. The decomposition of urea and urea derivatives is complex. In general, high-temperature decomposition of urea generates isocyanic acid and biuret, which can be converted to $\mathrm{CO}_{2}$ and $\mathrm{NH}_{3}$, or back to urea, respectively. ${ }^{78,79}$

3.4. Microstructure. The chitosan-urea aerogels presented here display a particle-network type microstructure (Figure 6), which is somewhat unusual as (nano) fibrillary structures are more common for chitosan aerogels. ${ }^{31,55}$ Both the SCD and APD aerogels consist of linked particles of $\sim 50$ $\mathrm{nm}$ in diameter (ranging from 20 to $90 \mathrm{~nm}$ ), corresponding to a theoretical surface area of $\sim 75 \mathrm{~m}^{2} / \mathrm{g}$ (ranging from 190 to 40 $\mathrm{m}^{2} / \mathrm{g}$ ), calculated under the assumption of spherical particles and a skeletal density of $1.59 \mathrm{~g} / \mathrm{cm}^{3}$ (Supporting Information) and consistent with those determined by nitrogen sorption analysis $\left(80-230 \mathrm{~m}^{2} / \mathrm{g}\right.$, Table 1$)$. The material displays local density variations between regions with predominantly mesoporous domains separated by macropores. The largest observed pores are 150 and $650 \mathrm{~nm}$ in diameter for the SCD(Figure 6a,c) and APD (Figure 6b,d)-dried aerogels, respectively. The higher macroporosity for the APD samples is related to platelet-like structures that were most likely 

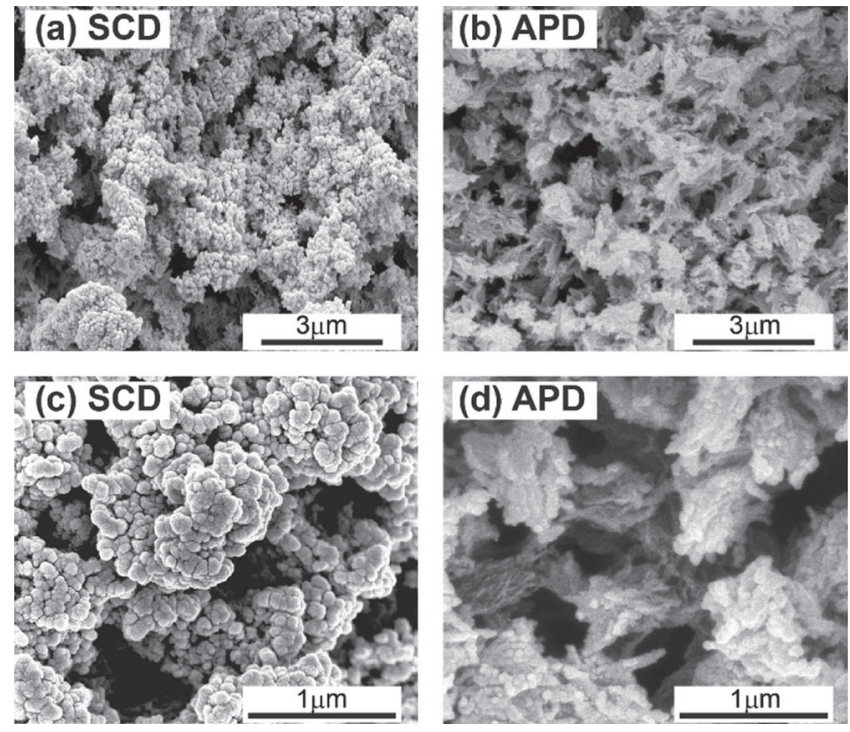

Figure 6. SEM images of chitosan-urea aerogels. (a) SCD aerogel $(30,000 \times)$. (b) APD aerogel $(30,000 \times)$. (c) SCD aerogel $(100,000 \times)$. (d) APD aerogel $(100,000 \times)$. SCD and APD images are from gels prepared from a 5 and $10 \% \mathrm{~m} / \mathrm{v}$ chitosan solution, respectively.

formed by a partial structural collapse during evaporative drying (Figure 6b,d).

Nitrogen sorption analysis further confirms the presence of a significant surface area and mesoporosity (Figure 7). All samples (SCD, APD RT, and APD $65^{\circ} \mathrm{C}$ ) display nitrogen sorption isotherms with a type-IV hysteresis, according to the IUPAC classification. The strong increase of the volume of $\mathrm{N}_{2}$ adsorbed in the capillary condensation regime, combined with a narrow desorption hysteresis loop is characteristic of the material's mesoporosity (Figures 7a, S3). This can also be observed in the BJH pore size distributions (Figures $7 \mathrm{~b}, \mathrm{~S} 3$ ), which are broad and include significant meso- and macroporosity for all samples. As is typical for aerogels, the BJHderived pore sizes are significantly lower than those derived from the envelope and skeletal density because of the inability for nitrogen sorption to sample larger pores, and the possible deformation of the aerogels upon desorption. ${ }^{57}$ The APD samples dried at room temperature display the highest densities and lowest surface areas and significant scatter in both density and surface area (Figure 7c). The APD samples dried at $65{ }^{\circ} \mathrm{C}$ give more consistent results and have intermediate densities and the highest surface areas. Finally, the SCD aerogels display the lowest densities and intermediate surface areas. The surface areas of the APD chitosan aerogels are in the same range as those for SCD, particularly for those dried at $65{ }^{\circ} \mathrm{C}$ (Figure 7c), in contrast to light-weight cellulose xerogels, where APD leads to a substantial decrease in the surface area. ${ }^{80}$

3.5. Mechanical Properties and Machinability. Regardless of the chitosan concentration, all aerogels prepared in this study sustain compression stress without rupture up to at least $80 \%$, and display relatively high $E$ moduli and $\sigma_{80}$ (Figure 8). Not surprisingly, the best mechanical performance is found for the densest aerogels, prepared with $12 \%$ chitosan, with a compressive $E$ modulus of $11.6 \mathrm{MPa}$ (Table 2). The samples are not brittle and no dust release is observed. The samples can be compressed up to $80 \%$ strain without rupture, with a $\sigma_{80}$ value as high as $17.9 \mathrm{MPa}$ (for $12 \%$ chitosan). However, the compression is mostly irreversible, even at smaller deformations, and the samples appear as cohesive, compressed, and flattened disks after testing and material properties such as density and thermal conductivity will start to deteriorate at much lower loads than the reported $\sigma_{80}$ values (Figure 8c). As is typical for aerogels, the $E$-modulus displays a power-law dependence on density $E \approx \rho^{\alpha}$, with $\alpha=2.6$, that is a linear dependence on density on a log-log plot (Figure $8 \mathrm{~b}$ ). Overall, the mechanical properties are in line with those observed for other biopolymer and biopolymer-silica hybrid aerogels, ${ }^{6,75,81-84}$ but much better than traditional silica aerogels. ${ }^{73}$ Remarkably, and in stark contrast to many other aerogel systems, both the SCD and APD aerogels are nonbrittle and machinable with standard tools. A variety of samples prepared from different chitosan concentrations and dried by both SCD and APD were machined by drilling and sawing. Systematic tests were carried out only for samples prepared from $10 \%$, both for SCD and APD. The samples resisted these tests without fracture (Figure 9).

3.6. Comparison to Other Biopolymer Aerogels. Biopolymer aerogels prepared by freeze drying (FD) most often display low surface areas and (very) low envelope densities, whereas those prepared by SCD display higher surface areas and a wide range in density (Figure 10a). Compared to other biopolymer aerogels prepared by SCD, the chitosan-urea SCD aerogels display moderately high surface areas and rather high envelope densities. Far fewer data have been reported for biopolymer aerogels prepared by APD, but the chitosan-urea APD aerogels presented here display
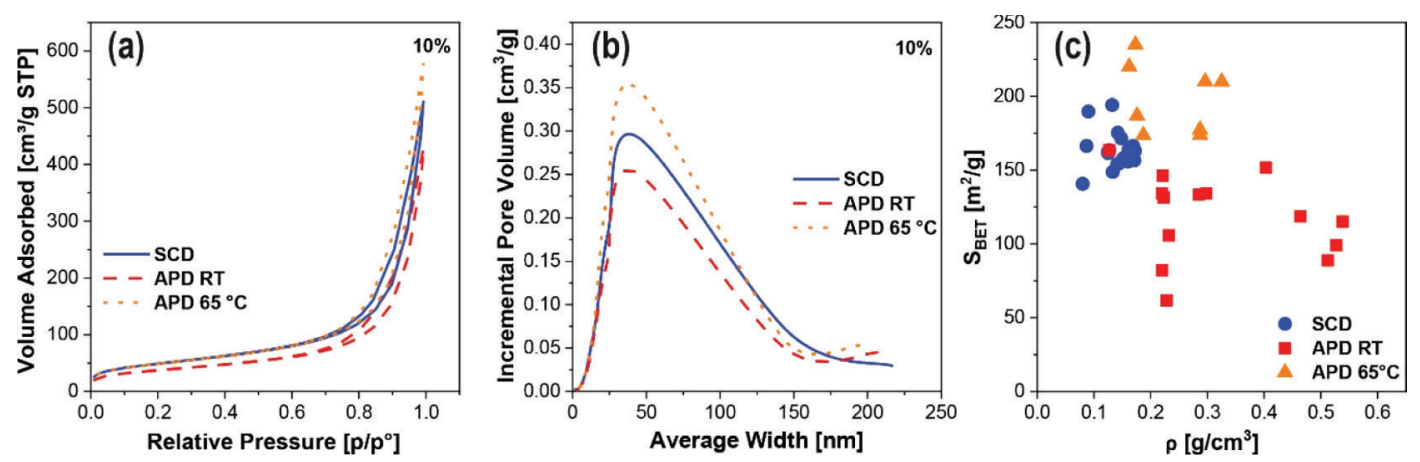

Figure 7. Nitrogen sorption data. (a) Nitrogen sorption isotherms of the $10 \%$ chitosan sample; (b) BJH desorption plots of the $10 \%$ chitosan sample; (c) Surface area as a function of density for all different chitosan concentrations. Isotherms and pore size distributions for other chitosan concentrations are shown in Figure S3. 

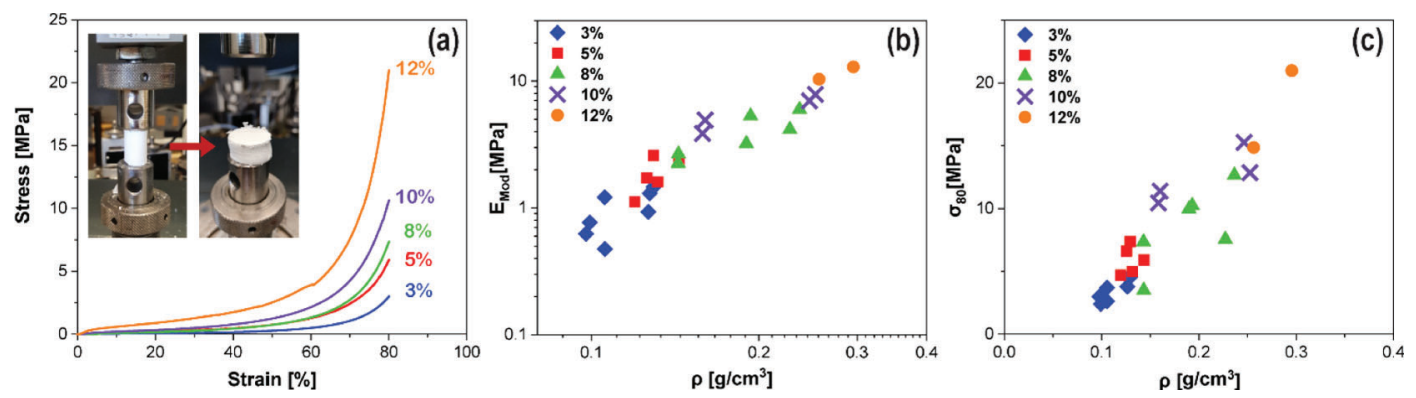

Figure 8. (a) Stress-strain curves of the SCD samples for different chitosan concentrations. (b) E modulus as a function of density (log-log plot). (c) Stress at $80 \%$ strain.

Table 2. Mechanical Properties of Chitosan Aerogels ${ }^{a}$

\begin{tabular}{|c|c|c|c|c|c|c|c|c|}
\hline & \multicolumn{3}{|c|}{$\rho\left[\mathrm{g} / \mathrm{cm}^{3}\right]$} & \multicolumn{2}{|c|}{$E[\mathrm{MPa}]$} & \multicolumn{2}{|c|}{${ }_{80}[\mathrm{MPa}]$} & \multirow[b]{2}{*}{$N$} \\
\hline & Chit. [\%] & Av. & SD & Av. & SD & Av. & SD & \\
\hline \multirow[t]{5}{*}{ SCD Aerogels } & $3 \%$ & 0.113 & 0.013 & 1.0 & 0.3 & 3.5 & 0.8 & 7 \\
\hline & $5 \%$ & 0.130 & 0.008 & 1.9 & 0.5 & 5.9 & 1.0 & 5 \\
\hline & $8 \%$ & 0.189 & 0.036 & 3.9 & 1.4 & 8.5 & 2.9 & 6 \\
\hline & $10 \%$ & 0.204 & 0.045 & 5.9 & 1.6 & 12.5 & 1.8 & 4 \\
\hline & $12 \%$ & 0.276 & 0.020 & 11.6 & 1.3 & 17.9 & 3.1 & 2 \\
\hline
\end{tabular}

${ }^{a} \mathrm{SD}$ : standard deviation from $\mathrm{N}$ measurements on $N$ different samples $\left(\sqrt{\frac{\sum(x-\bar{x})^{2}}{N}}\right)$.
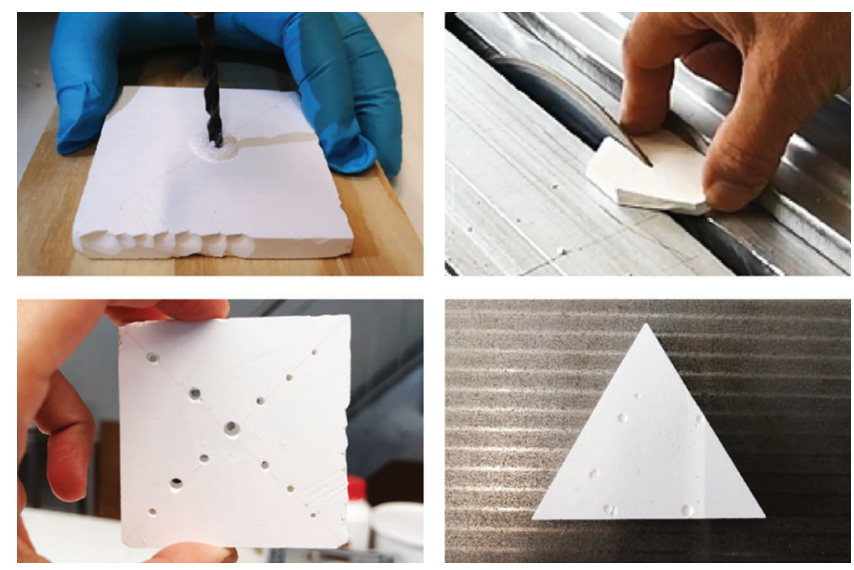

Figure 9. Machinability of the $10 \%$ chitosan aerogels and xerogels subjected to drilling and sawing.

relatively high surface areas, particularly those dried at $65{ }^{\circ} \mathrm{C}$ (Figure 10a).

The last few years have seen a rapid increase in the reported thermal conductivity data for biopolymer aerogels (Figure $10 \mathrm{~b})$. Biopolymer aerogels with thermal conductivities below $20 \mathrm{~mW} /(\mathrm{m} \cdot \mathrm{K})$ cluster around densities of $0.100 \mathrm{~g} / \mathrm{cm}^{3}$ and surface areas above $300 \mathrm{~m}^{2} / \mathrm{g}$, and all have been prepared by supercritical drying. A minimum in thermal conductivity at intermediate densities is typical for aerogels: at higher densities, heat conduction through the solid skeleton increases rapidly; at low densities, there is not enough material available to partition the space into small enough pores to suppress the gas phase conduction by the Knudsen effect. ${ }^{1}$ Notable examples of superinsulating aerogels in this intermediate density range include pectin, ${ }^{85}$ cellulose, ${ }^{86}$ and chitosan aerogels. ${ }^{31}$ The SCD chitosan-urea aerogels from this study display thermal conductivities below $26 \mathrm{~mW} /(\mathrm{m} \cdot \mathrm{K})$ (standing air) and well below $30-35 \mathrm{~mW} /(\mathrm{m} \cdot \mathrm{K})$ (mineral wool, EPS), indicative of a partial suppression of the gas phase conduction and consistent with the estimated pore sizes (Figure 10b). The thermal conductivity is, however, above $20 \mathrm{~mW} /(\mathrm{m} \cdot \mathrm{K})$, most likely because of the relatively high densities, the only moderately high surface areas, and the only moderately small pore sizes. Thus, a reduction in thermal conductivity will require further optimization toward lower densities and/or higher surface areas.

The APD chitosan-urea aerogels are the first APD biopolymer aerogels for which thermal conductivity data are reported. They display thermal conductivities as low as 30.9 $\mathrm{mW} /(\mathrm{m} \cdot \mathrm{K})$, that is lower than natural insulation materials (e.g. wool or stray) and at the lower end of what is possible with conventional, air-filled insulation materials (e.g. mineral wool or EPS), albeit with very different densities and pore structures. Given the estimated pore size, the gas-phase conduction is expected to be partially reduced for the APD chitosan-urea aerogels, but not for conventional insulators. However, these benefits are negated by the higher densities of 0.167 to $0.304 \mathrm{~g} / \mathrm{cm}^{3}$ compared to 0.015 to $0.050 \mathrm{~g} / \mathrm{cm}^{3}$ for conventional insulators. With these similar thermal conductivity values, APD chitosan-urea aerogels are not competitive with inexpensive, conventional insulation products. However, the APD chitosan-urea aerogels provide the first demonstration that high-performance biopolymer aerogel insulation produced by ambient pressure drying may be within reach, a feat that for now remains exclusive to silica aerogels.

\section{CONCLUSIONS}

Sustainable biopolymer aerogels are produced from renewable, waste-derived chitosan and urea as a "green" modifier. The reaction of chitosan with urea in an aqueous medium grafts abundant ureido groups onto the chitosan backbone, possibly in addition to minor cross-linking ureylene. This modification alters the chitosan-chitosan and chitosan-solvent interactions and leads to irreversible gelation, without the need for toxic cross-linkers such as formaldehyde. The urea-modified chitosan aerogels display exceptional mechanical properties 
a

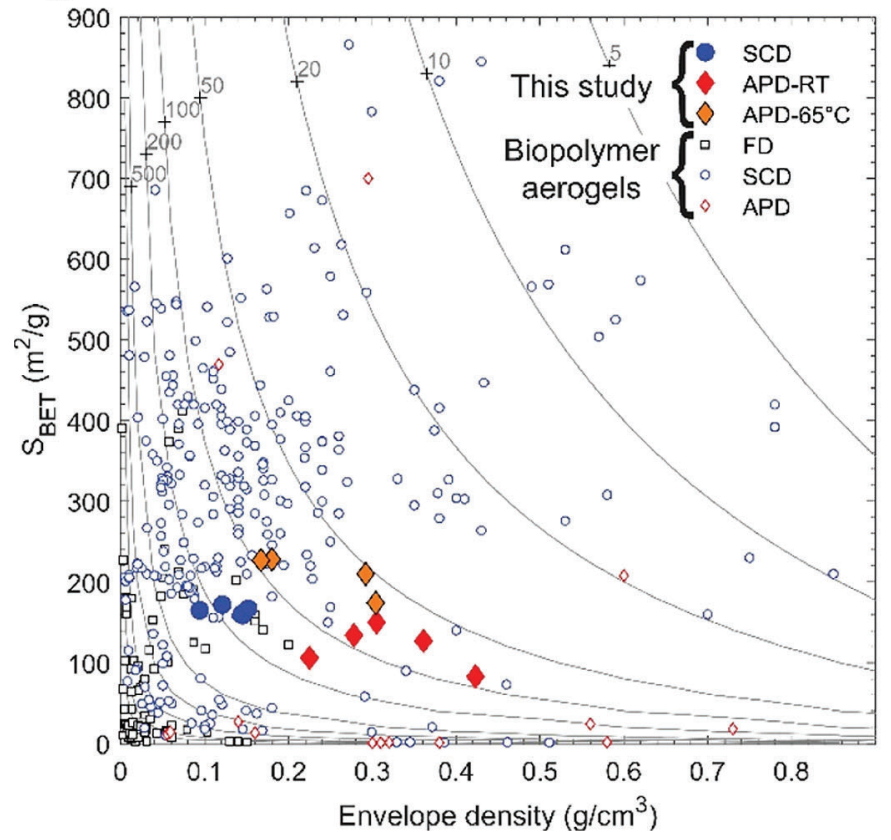

b

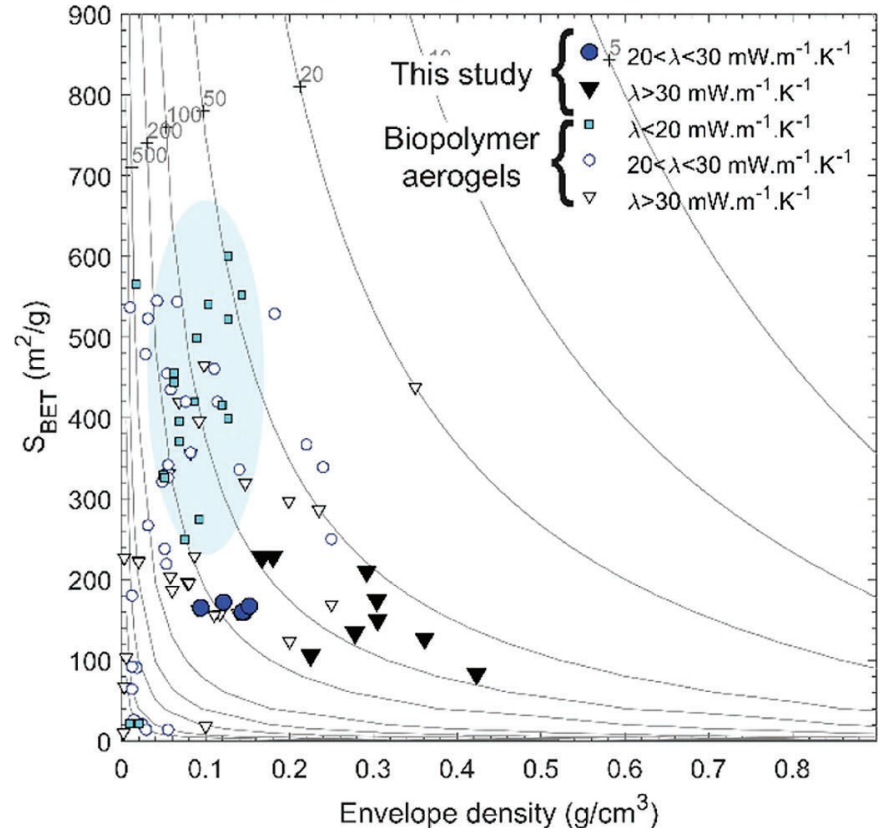

Figure 10. Comparison to other biopolymer aerogels. (a) Surface area vs envelope density, separated using the drying technique. (b) Surface area versus envelope density, separated by thermal conductivity. The contour lines denote average pore sizes (in nm) calculated from density and surface area, assuming cylindrical pores. Data source: 2018 review paper $^{6}$ augmented with more recent literature data (Table S11).

(no brittle rupture and easy machinability) and moderately high surface area and mesoporosity. The thermal conductivity reaches values below that of standing air or conventional insulation materials. We also find a remarkably high-temperature stability and self-extinguishing behavior during simplified fire testing. The simple and nontoxic synthesis process and excellent machinability broaden their application potential, for example toward insulation and biomedical applications. Remarkably, this class of chitosan-urea gels can be transformed into mesoporous, monolithic aerogels by evaporative drying. The ability to produce monolithic, mesoporous chitosan aerogels by ambient pressure drying, a first in the field of biopolymer aerogels, represents an important milestone toward cost-effective biopolymer aerogel production.

\section{ASSOCIATED CONTENT}

\section{OSupporting Information}

The Supporting Information is available.

Effect of different reagent concentrations, chitosan: urea ratio, temperature, time, $\mathrm{HCl}$ concentration, chitosan molecular weight on gelation, and aerogel properties, chitosan solubility in different acids, tables with peak positions and assignment of the solid-state NMR and FTIR spectra, calculations of the theoretical relationship between the surface area and particle size, humidity uptake and nitrogen sorption data for different chitosan concentrations, flame test on the SCD samples, thermal conductivity device, and theoretical estimate of ureylene versus ureido concentration (PDF)

Recent biopolymer aerogel data (XLSX)

\section{AUTHOR INFORMATION}

\section{Corresponding Authors}

Shanyu Zhao - Laboratory for Building Energy Materials and Components, Swiss Federal Laboratories for Materials Science and Technology, Empa, CH-8600 Dübendorf, Switzerland; Phone: +41 58765 4244; Email: shanyu.zhao@empa.ch

Wim J. Malfait - Laboratory for Building Energy Materials and Components, Swiss Federal Laboratories for Materials Science and Technology, Empa, CH-8600 Dübendorf, Switzerland; ○ orcid.org/0000-0002-1668-8749; Phone: +41 58765 4983; Email: wim.malfait@empa.ch

\section{Authors}

Natalia Guerrero-Alburquerque - Laboratory for Building Energy Materials and Components, Swiss Federal Laboratories for Materials Science and Technology, Empa, CH-8600 Dübendorf, Switzerland; Department of Chemistry, University of Fribourg, CH-1700 Fribourg, Switzerland

Nour Adilien - Laboratory for Building Energy Materials and Components, Swiss Federal Laboratories for Materials Science and Technology, Empa, CH-8600 Dübendorf, Switzerland

Matthias M. Koebel - Laboratory for Building Energy Materials and Components, Swiss Federal Laboratories for Materials Science and Technology, Empa, CH-8600 Dübendorf, Switzerland

Marco Lattuada - Department of Chemistry, University of Fribourg, CH-1700 Fribourg, Switzerland; (1) orcid.org/00000001-7058-9509

Complete contact information is available at: https://pubs.acs.org/10.1021/acsami.0c03047

\section{Notes}

The authors declare no competing financial interest. 


\section{ACKNOWLEDGMENTS}

We thank Beatrice Fischer for assistance with FTIR, mechanical testing, and TGA measurements, Daniel Rentsch for his assistance and suggestions with NMR measurements, Robin Pauer for help with the SEM imaging, Sara Aleksandra Madry for help with density measurements, Samuel Brunner for support with the guarded hotplate measurements, and Gudrun Reichenauer for test measurements of thermal conductivity on preliminary samples. This work was supported by the Swiss National Foundation through grant 200021_179000 (W.J.M.).

\section{REFERENCES}

(1) Hüsing, N.; Schubert, U. Aerogels-Airy Materials: Chemistry, Structure, and Properties. Angew. Chem., Int. Ed. 1998, 37, 22-45.

(2) Aegerter, M.; Leventis, N.; Koebel, M. Aerogels Handbook; Aegerter, M. A., Leventis, N., Koebel, M. M., Eds.; Springer New York Dordrecht Heidelberg London: New York, NY, 2011.

(3) Koebel, M.; Rigacci, A.; Achard, P. Aerogel-Based Thermal Superinsulation: An Overview. J. Sol-Gel Sci. Technol. 2012, 63, 315339.

(4) Baetens, R.; Jelle, B. P.; Gustavsen, A. Aerogel Insulation for Building Applications: A State-of-the-Art Review. Energy Build. 2011, 43, 761-769.

(5) Prakash, S. S.; Brinker, C. J.; Hurd, A. J.; Rao, S. M. Silica Aerogel Films Prepared at Ambient Pressure by Using Surface Derivatization to Induce Reversible Drying Shrinkage. Nature 1995, 374, 439-443.

(6) Zhao, S.; Malfait, W. J.; Guerrero-Alburquerque, N.; Koebel, M. M.; Nyström, G. Biopolymer Aerogels and Foams: Chemistry, Properties, and Applications. Angew. Chem., Int. Ed. 2018, 57, $7580-7608$.

(7) Yan, N.; Chen, X. Sustainability: Don't Waste Seafood Waste. Nature 2015, 524, 155-157.

(8) Hülsey, M. J. Shell Biorefinery: A Comprehensive Introduction. Green Energy Environ. 2018, 3, 318-327.

(9) Risen, W. M.; Ji, S.; Hu, X.; Zhang, R. Aerogel Materials And Detectors, Liquid And Gas Absorbing Objects, And Optical Devices Comprising Same. U.S. Patent 6,303,046 B1, 2001.

(10) Alhwaige, A. A.; Agag, T.; Ishida, H.; Qutubuddin, S. Biobased Chitosan Hybrid Aerogels with Superior Adsorption: Role of Graphene Oxide in CO2 Capture. RSC Adv. 2013, 3, 16011-16020. (11) Varma, A. J.; Deshpande, S. V.; Kennedy, J. F. Metal Complexation by Chitosan and Its Derivatives: A Review. Carbohydr. Polym. 2004, 55, 77-93.

(12) Guibal, E. Interactions of Metal Ions with Chitosan-Based Sorbents: A Review. Sep. Purif. Technol. 2004, 38, 43-74.

(13) Zhang, S.; Feng, J.; Feng, J.; Jiang, Y. Formation of Enhanced Gelatum Using Ethanol/Water Binary Medium for Fabricating Chitosan Aerogels with High Specific Surface Area. Chem. Eng. J. 2017, 309, 700-707.

(14) Wang, J.; Zhou, Q.; Song, D.; Qi, B.; Zhang, Y.; Shao, Y.; Shao, Z. Chitosan-Silica Composite Aerogels: Preparation, Characterization and Congo Red Adsorption. J. Sol-Gel Sci. Technol. 2015, 76, 501-509.

(15) Chang, X.; Chen, D.; Jiao, X. Chitosan-Based Aerogels with High Adsorption Performance. J. Phys. Chem. B 2008, 112, 77217725 .

(16) Mahaninia, M. H.; Wilson, L. D. Cross-Linked Chitosan Beads for Phosphate Removal from Aqueous Solution. J. Appl. Polym. Sci. 2015, 133, 42949.

(17) Chtchigrovsky, M.; Primo, A.; Gonzalez, P.; Molvinger, K.; Robitzer, M.; Quignard, F.; Taran, F. Functionalized Chitosan as a Green, Recyclable, Biopolymer-Supported Catalyst for the $[3+2]$ Huisgen Cycloaddition. Angew. Chem., Int. Ed. 2009, 48, 5916-5920. (18) Kadib, A. E.; Molvinger, K.; Cacciaguerra, T.; Bousmina, M.; Brunel, D. Chitosan Templated Synthesis of Porous Metal Oxide
Microspheres with Filamentary Nanostructures. Microporous Mesoporous Mater. 2011, 142, 301-307.

(19) Frindy, S.; Primo, A.; Lahcini, M.; Bousmina, M.; Garcia, H.; El Kadib, A. Pd Embedded in Chitosan Microspheres as Tunable SoftMaterials for Sonogashira Cross-Coupling in Water-Ethanol Mixture. Green Chem. 2015, 17, 1893-1898.

(20) Frindy, S.; Primo, A.; Ennajih, H.; el kacem Qaiss, A.; Bouhfid, R.; Lahcini, M.; Essassi, E. M.; Garcia, H.; El Kadib, A. ChitosanGraphene Oxide Films and CO2-Dried Porous Aerogel Microspheres: Interfacial Interplay and Stability. Carbohydr. Polym. 2017, 167, 297305.

(21) Primo, A.; Quignard, F. Chitosan as Efficient Porous Support for Dispersion of Highly Active Gold Nanoparticles: Design of Hybrid Catalyst for Carbon-Carbon Bond Formation. Chem. Commun. 2010, 46, 5593.

(22) Quignard, F.; Valentin, R.; Di Renzo, F. Aerogel Materials from Marine Polysaccharides. New J. Chem. 2008, 32, 1300.

(23) Kayser, H.; Müller, C. R.; García-González, C. A.; Smirnova, I.; Leitner, W.; Domínguez de María, P. Dried Chitosan-Gels as Organocatalysts for the Production of Biomass-Derived Platform Chemicals. Appl. Catal., A 2012, 445-446, 180-186.

(24) Hu, X.; Littrel, K.; Ji, S.; Pickles, D. G.; Risen, W. M. Characterization of Silica-Polymer Aerogel Composites by SmallAngle Neutron Scattering and Transmission Electron Microscopy. J. Non-Cryst. Solids 2001, 288, 184-190.

(25) Yao, C.; Risen, W. M. Formation and Reaction of MetalContaining Nanoparticles in Organic/Inorganic Hybrid Aerogels. MRS Proc. 2005, 847, EE5.10.1-EE.10.6.

(26) Singh, J.; Dutta, P. K.; Dutta, J.; Hunt, A. J.; Macquarrie, D. J.; Clark, J. H. Preparation and Properties of Highly Soluble Chitosan-lGlutamic Acid Aerogel Derivative. Carbohydr. Polym. 2009, 76, 188195.

(27) Wang, M.; Liu, X.; Ji, S.; Risen, W. M. A New Hybrid Aerogel Approach to Modification of Bioderived Polymers for Materials Applications. MRS Proc. 2002, 702, U3.5.1-U3.5.9.

(28) Zhao, S.; Xu, H.; Wang, L.; Zhu, P.; Risen, W. M.; William Suggs, J. Synthesis of Novel Chitaline-Silica Aerogels with Spontaneous Au and Ag Nanoparticles Formation in Aerogels Matrix. Microporous Mesoporous Mater. 2013, 171, 147-155.

(29) Wang, R.; Shou, D.; Lv, O.; Kong, Y.; Deng, L.; Shen, J. PHControlled Drug Delivery with Hybrid Aerogel of Chitosan, Carboxymethyl Cellulose and Graphene Oxide as the Carrier. Int. J. Biol. Macromol. 2017, 103, 248-253.

(30) Iglesias, C. L.; Barros, J.; Ardao, I.; Gurikov, P.; Monteiro, F. J.; Smirnova, I.; Lorenzo, C. A.; González, C. A. G. Jet Cutting Technique for the Production of Chitosan Aerogel Microparticles Loaded with Vancomycin. Polymers 2020, 1-13, 273.

(31) Takeshita, S.; Yoda, S. Chitosan Aerogels: Transparent, Flexible Thermal Insulators. Chem. Mater. 2015, 27, 7569-7572.

(32) Takeshita, S.; Yoda, S. Translucent, Hydrophobic, and Mechanically Tough Aerogels Constructed from Trimethylsilylated Chitosan Nanofibers. Nanoscale 2017, 9, 12311-12315.

(33) Zhao, S.; Malfait, W. J.; Jeong, E.; Fischer, B.; Zhang, Y.; Xu, H.; Angelica, E.; Risen, W. M.; Suggs, J. W.; Koebel, M. M. Facile One-Pot Synthesis of Mechanically Robust Biopolymer-Silica Nanocomposite Aerogel by Cogelation of Silicic Acid with Chitosan in Aqueous Media. ACS Sustainable Chem. Eng. 2016, 4, 5674-5683.

(34) El Kadib, A.; Bousmina, M. Chitosan Bio-Based OrganicInorganic Hybrid Aerogel Microspheres. Chem.-Eur. J. 2012, 18, 8264-8277.

(35) Wang, Q. Z.; Chen, X. G.; Liu, N.; Wang, S. X.; Liu, C. S.; Meng, X. H.; Liu, C. G. Protonation Constants of Chitosan with Different Molecular Weight and Degree of Deacetylation. Carbohydr. Polym. 2006, 65, 194-201.

(36) Raman, S. P.; Gurikov, P.; Smirnova, I. Hybrid Alginate Based Aerogels by Carbon Dioxide Induced Gelation: Novel Technique for Multiple Applications. J. Supercrit. Fluids 2015, 106, 23-33.

(37) Iglesias, N.; Galbis, E.; Valencia, C.; de-Paz, M.-V.; Galbis, J. Reversible PH-Sensitive Chitosan-Based Hydrogels. Influence of 
Dispersion Composition on Rheological Properties and Sustained Drug Delivery. Polymer 2018, 10, 392.

(38) Nilsen-Nygaard, J.; Strand, S.; Vårum, K.; Draget, K.; Nordgård, C. Chitosan: Gels and Interfacial Properties. Polymer 2015, 7, 552-579.

(39) Cho, J.; Heuzey, M.-C.; Bégin, A.; Carreau, P. J. Physical Gelation of Chitosan in the Presence of $\beta$-Glycerophosphate: The Effect of Temperature. Biomacromolecules 2005, 6, 3267-3275.

(40) Salam, A.; Venditti, R. A.; Pawlak, J. J.; El-Tahlawy, K. Crosslinked Hemicellulose Citrate-Chitosan Aerogel Foams. Carbohydr. Polym. 2011, 84, 1221-1229.

(41) Ganesan, K.; Budtova, T.; Ratke, L.; Gurikov, P.; Baudron, V.; Preibisch, I.; Niemeyer, P.; Smirnova, I.; Milow, B. Review on the Production of Polysaccharide Aerogel Particles. Materials 2018, 11, 2144.

(42) Gavillon, R.; Budtova, T. Aerocellulose: New Highly Porous Cellulose Prepared from Cellulose- $\mathrm{NaOH}$ Aqueous Solutions. Biomacromolecules 2008, 9, 269-277.

(43) Tkalec, G.; Knez, Ž.; Novak, Z. Formation of Polysaccharide Aerogels in Ethanol. RSC Adv. 2015, 5, 77362-77371.

(44) Takeshita, S.; Sadeghpour, A.; Malfait, W. J.; Konishi, A.; Otake, K.; Yoda, S. Formation of Nanofibrous Structure in Biopolymer Aerogel during Supercritical CO 2 Processing: The Case of Chitosan Aerogel. Biomacromolecules 2019, 20, 2051-2057.

(45) Takeshita, S.; Konishi, A.; Takebayashi, Y.; Yoda, S.; Otake, K. Aldehyde Approach to Hydrophobic Modification of Chitosan Aerogels. Biomacromolecules 2017, 18, 2172-2178.

(46) G, S.; Mitra, T.; Chatterjee, S.; Gnanamani, A. Chemistry behind the Elastic Nature of the Biomaterial Prepared Using Oxidized Form of Glutaraldehyde and Chitosan - an Approach at 2D and 3D Level. Int. J. Life Sci. Med. Res. 2013, 3, 64-75.

(47) Baldino, L.; Concilio, S.; Cardea, S.; De Marco, I.; Reverchon, E. Complete Glutaraldehyde Elimination during Chitosan Hydrogel Drying by SC-CO2 Processing. J. Supercrit. Fluids 2015, 103, 70-76. (48) Shang, J.; Liu, S.; Ma, X.; Lu, L.; Deng, Y. A New Route of CO2 Catalytic Activation: Syntheses of N-Substituted Carbamates from Dialkyl Carbonates and Polyureas. Green Chem. 2012, 14, 2899-2906.

(49) Carretti, E.; Dei, L.; Baglioni, P.; Weiss, R. G. Synthesis and Characterization of Gels from Polyallylamine and Carbon Dioxide as Gellant. J. Am. Chem. Soc. 2003, 125, 5121-5129.

(50) Markiewitz, K. H.; Wilmington, D. Polymers Preapred by Reacting Urea with an Amino Alcohol or Diamine Followed by Methylolation with Formaldehyde. U.S. Patent 3,763,106 A, 1973.

(51) Arnold, H. W. Process of Making Aliphatic Polyureas. U.S. Patent 2,145,242 A, 1939.

(52) Gabler, R.; Müller, H. Preparation of Linear Polyureas Utilizing a Cyclic Amide Solvent. U.S. Patent 3,185,656 A, 1965.

(53) Chenite, A.; Gori, S.; Shive, M.; Desrosiers, E.; Buschmann, M. D. Monolithic Gelation of Chitosan Solutions via Enzymatic Hydrolysis of Urea. Carbohydr. Polym. 2006, 64, 419-424.

(54) Krajewska, B. A Combined Temperature-PH Study of Urease Kinetics. Assigning PKavalues to Ionizable Groups of the Active Site Involved in the Catalytic Reaction. J. Mol. Catal. B Enzym. 2016, 124, $70-76$.

(55) Ganesan, K.; Heyer, M.; Ratke, L.; Milow, B. Facile Preparation of Nanofibrillar Networks of "Ureido-Chitin" Containing Ureido and Amine as Chelating Functional Groups. Chem.-Eur. J. 2018, 24, 19332-19340.

(56) Barrett, E. P.; Joyner, L. G.; Halenda, P. P. The Determination of Pore Volume and Area Distributions in Porous Substances. I. Computations from Nitrogen Isotherms. Am. Chem. Soc. 1951, 73, 373.

(57) Reichenauer, G. Structural Characterization of Aerogels. In Aerogels Handbook; Aegerter, M. A., Leventis, N., Koebel, M. M., Eds.; Springer New York: New York, NY, 2011; pp 449-498.

(58) Stahl, T.; Brunner, S.; Zimmermann, M.; Ghazi Wakili, K. Thermo-Hygric Properties of a Newly Developed Aerogel Based
Insulation Rendering for Both Exterior and Interior Applications. Energy Build. 2012, 44, 114-117.

(59) Barzagli, F.; Mani, F.; Peruzzini, M. From Greenhouse Gas to Feedstock: Formation of Ammonium Carbamate from $\mathrm{CO} 2$ and $\mathrm{NH} 3$ in Organic Solvents and Its Catalytic Conversion into Urea under Mild Conditions. Green Chem. 2011, 13, 1267-1274.

(60) Alexandrova, A. N.; Jorgensen, W. L. Why Urea Eliminates Ammonia Rather than Hydrolyzes in Aqueous Solution. J. Phys. Chem. B 2007, 111, 720-730.

(61) Mani, F.; Peruzzini, M.; Stoppioni, P. CO 2 Absorption by Aqueous NH 3 Solutions: Speciation of Ammonium Carbamate, Bicarbonate and Carbonate by a $13 \mathrm{C}$ NMR Study. Green Chem. 2006, 8, 995-1000.

(62) Matsuzaki, Y.; Yamada, H.; Chowdhury, F. A.; Higashii, T.; Kazama, S.; Onoda, M. Ab Initio Study of CO2 Capture Mechanisms in Monoethanolamine Aqueous Solution: Reaction Pathways from Carbamate to Bicarbonate. Energy Procedia 2013, 37, 400-406.

(63) Yamamoto, Y.; Hasegawa, J.-y.; Ito, Y. Kinetic Investigation on Carbamate Formation from the Reaction of Carbon Dioxide with Amino Acids in Homogeneous Aqueous Solution. J. Phys. Org. Chem. 2012, 25, 239-247.

(64) Wang, X.; Conway, W.; Fernandes, D.; Lawrance, G.; Burns, R.; Puxty, G.; Maeder, M. Kinetics of the Reversible Reaction of CO2(Aq) with Ammonia in Aqueous Solution. J. Phys. Chem. A 2011, $115,6405-6412$.

(65) Walker, J.; Hambly, F. J. LXXVII.-Transformation of Ammonium Cyanate into Urea. J. Chem. Soc., Faraday Trans. 1895, $67,746-767$

(66) Yim, S. D.; Kim, S. J.; Baik, J. H.; Nam, I. S.; Mok, Y. S.; Lee, J.H.; Cho, B. K.; Oh, S. H. Decomposition of Urea into NH 3 for the SCR Process. Ind. Eng. Chem. Res. 2004, 43, 4856-4863.

(67) Einbu, A.; Vårum, K. M. Depolymerization and De-NAcetylation of Chitin Oligomers in Hydrochloric Acid. Biomacromolecules 2007, 8, 309-314.

(68) Kasaai, M. R.; Arul, J.; Charlet, G. Fragmentation of Chitosan by Acids. J. Sci. World 2013, 2013, 508540.

(69) Marji, T. J. Separation and Purification of Hydrophilic Polymers. M.Sc Thesis, Pharmaceutical Technology 263467894, Jordan University of Science and Technology, 2008.

(70) Mattia, J.; Painter, P. A Comparison of Hydrogen Bonding and Order in a Polyurethane and Poly(Urethane-Urea) and Their Blends with Poly(Ethylene Glycol). Macromolecules 2007, 40, 1546-1554.

(71) Russo, P. S. A Perspective on Reversible Gels and Related Systems; American Chemical Society, 1987; pp 1-21.

(72) Gurikov, P.; Raman, S. P.; Steiner, S. A., III; Smirnova, I. 110th Anniversary: Solvent Exchange in the Processing of Biopolymer Aerogels: Current Status and Open Questions. Ind. Eng. Chem. Res. 2019, 58, 18590.

(73) Wong, J. C. H.; Kaymak, H.; Brunner, S.; Koebel, M. M. Mechanical Properties of Monolithic Silica Aerogels Made from Polyethoxydisiloxanes. Microporous Mesoporous Mater. 2014, 183, $23-$ 29.

(74) Abdou, A.; Budaiwi, I. The Variation of Thermal Conductivity of Fibrous Insulation Materials under Different Levels of Moisture Content. Constr. Build. Mater. 2013, 43, 533-544.

(75) Zhao, S.; Malfait, W. J.; Demilecamps, A.; Zhang, Y.; Brunner, S.; Huber, L.; Tingaut, P.; Rigacci, A.; Budtova, T.; Koebel, M. M. Strong, Thermally Superinsulating Biopolymer-Silica Aerogel Hybrids by Cogelation of Silicic Acid with Pectin. Angew. Chem., Int. Ed. 2015, 54, 14282-14286.

(76) Laufer, G.; Kirkland, C.; Cain, A. A.; Grunlan, J. C. ClayChitosan Nanobrick Walls: Completely Renewable Gas Barrier and Flame-Retardant Nanocoatings. ACS Appl. Mater. Interfaces 2012, 4, 1643-1649.

(77) Leistner, M.; Abu-Odeh, A. A.; Rohmer, S. C.; Grunlan, J. C. Water-Based Chitosan/Melamine Polyphosphate Multilayer Nanocoating That Extinguishes Fire on Polyester-Cotton Fabric. Carbohydr. Polym. 2015, 130, 227-232. 
(78) Bernhard, A. M. Catalytic Urea Decomposition, Side-Reactions and Urea Evaporation in the Selective Catalytic Reduction of NOx. Ph.D. Thesis, ETH, 2012.

(79) Todorova, T.; Peitz, D.; Kröcher, O.; Wokaun, A.; Delley, B. Guanidinium Formate Decomposition on the (101) TiO2-Anatase Surface: Combined Minimum Energy Reaction Pathway Calculations and Temperature-Programmed Decomposition Experiments. J. Phys. Chem. C 2011, 115, 1195-1203.

(80) Pour, G.; Beauger, C.; Rigacci, A.; Budtova, T. Xerocellulose: Lightweight, Porous and Hydrophobic Cellulose Prepared via Ambient Drying. J. Mater. Sci. 2015, 50, 4526-4535.

(81) Iswar, S.; Snellings, G. M. B. F.; Zhao, S.; Erni, R.; Bahk, Y. K.; Wang, J.; Lattuada, M.; Koebel, M. M.; Malfait, W. J. Reinforced and Superinsulating Silica Aerogel through in Situ Cross-Linking with Silane Terminated Prepolymers. Acta Mater. 2018, 147, 322-328.

(82) Rudaz, C.; Courson, R.; Bonnet, L.; Calas-Etienne, S.; Sallée, H.; Budtova, T. Aeropectin: Fully Biomass-Based Mechanically Strong and Thermal Superinsulating Aerogel. Biomacromolecules 2014, 15, 2188-2195.

(83) Demilecamps, A.; Beauger, C.; Hildenbrand, C.; Rigacci, A.; Budtova, T. Cellulose-Silica Aerogels. Carbohydr. Polym. 2015, 122, 293-300.

(84) Cheng, Y.; Lu, L.; Zhang, W.; Shi, J.; Cao, Y. Reinforced Low Density Alginate-Based Aerogels: Preparation, Hydrophobic Modification and Characterization. Carbohydr. Polym. 2012, 88, 10931099.

(85) Groult, S.; Budtova, T. Thermal Conductivity/Structure Correlations in Thermal Super-Insulating Pectin Aerogels. Carbohydr. Polym. 2018, 196, 73-81.

(86) Plappert, S. F.; Nedelec, J.-M.; Rennhofer, H.; Lichtenegger, H. C.; Liebner, F. W. Strain Hardening and Pore Size Harmonization by Uniaxial Densification: A Facile Approach toward Superinsulating Aerogels from Nematic Nanofibrillated 2,3-Dicarboxyl Cellulose. Chem. Mater. 2017, 29, 6630-6641. 